\title{
Using diamond as a metastable phase carbon source to facilitate the synthesis of graphite encapsulated metal (GEM) nanoparticles by an arc-discharge method
}

\author{
Mao-Hua Teng *, Shao-Wei Tsai, Chung-I Hsiao, Yung-Der Chen \\ No. 1, Sec. 4, Roosevelt Road, Department of Geosciences, National Taiwan University, Taiwan, ROC
}

Available online 16 October 2006

\begin{abstract}
Graphite encapsulated metal (GEM) nanocrystals have many potentially important applications, such as use in sensor devices, or as hydrogen storage materials. To improve the synthesis efficiency of ferromagnetic GEM (Fe, Co, and Ni), synthetic diamond was used as carbon source, replacing thermodynamically stable graphite, on a custom-designed tungsten arc-discharge device. The results show that diamond is a much better raw material than graphite; the new process not only raises the production rate (except for Co) of the as-made powder, but also increases the recovery percentage of GEM nanocrystals from the original 7-10\% to $23-33 \%$. The exact mechanism is still unclear, but some evidence indicates that diamond as a metastable phase of carbon probably dissolves in liquid metal pools during arcing much more easily than does the stable graphite. The experiments also provide valuable information on the phase transformation of diamond into graphite.
\end{abstract}

(C) 2006 Elsevier B.V. All rights reserved.

Keywords: Metals; Nanostructures; Vapor deposition; Scanning and transmission electron microscopy

\section{Introduction}

Graphite encapsulated metal (GEM) nanocrystals are composite materials with a distinctive spherical core-and-shell structure $(5-50 \mathrm{~nm}$ in diameter) [1-7]. The core is metal nanocrystal and the shell comprises of several to over 10 layers of graphitic sheets. Through the protection of the outer graphitic shell, the inner metal nanoparticles are stable in strong acid, even at high temperatures if in an oxygen-free environment. Among many types of GEM with various core materials, those with ferromagnetic metal cores are particularly interesting, not only because of their ferromagnetic properties, but also because the typical ferromagnetic metals $\mathrm{Fe}, \mathrm{Co}$, and $\mathrm{Ni}$ have a unique catalyzing ability to transform amorphous carbon into graphite [4,5].

GEM nanoparticles have many potentially important applications. For example, in micro-machinery, their spherical shape and outer graphitic shells make them an ideal candidate for nanoball bearings or lubricants. In fuel cells, some GEM materials have shown very promising hydrogen storage ability. Finally, in biomedical research, magnetic GEM particles can be an effective

\footnotetext{
* Corresponding author. Tel.: +886 23698316; fax: +886223636095.

E-mail address: mhteng@ntu.edu.tw (M.-H. Teng).
}

drug carrier that can be used to deliver specific doses of medicine to any part of the human body. However, before any such applications can become practical, it is necessary to improve our synthesis method in terms of both the amount of production and efficiency.

In our previous experiments, efficiency was improved greatly by using a custom-designed tungsten arc-discharge device [8]. However, the carbon source that is used on this device is graphite powder only, and a more efficient carbon source material is needed. As a metastable phase at ordinary T, P conditions, diamond is an obvious choice. Diamond is expected to more easily decompose (or dissolve) in liquid metal pool when arcing. Thus, when evaporation occurs, it should provide more carbon vapor in the synthesis of GEM. This argument, however, is not entirely correct, as will be shown in the later sections of this paper. In addition, for the main purpose of this paper, i.e., to decide whether diamond is a good carbon source material, the minor differences between $\mathrm{Fe}, \mathrm{Co}$, and $\mathrm{Ni}$ will not be discussed, and the experimental results in general will be concentrated on.

\section{Experimental}

In contrast to the popular carbon arc-discharge method (i.e., the KrätschmerHuffman method), a custom tungsten arc-discharge device (Fig. 1) was used to 


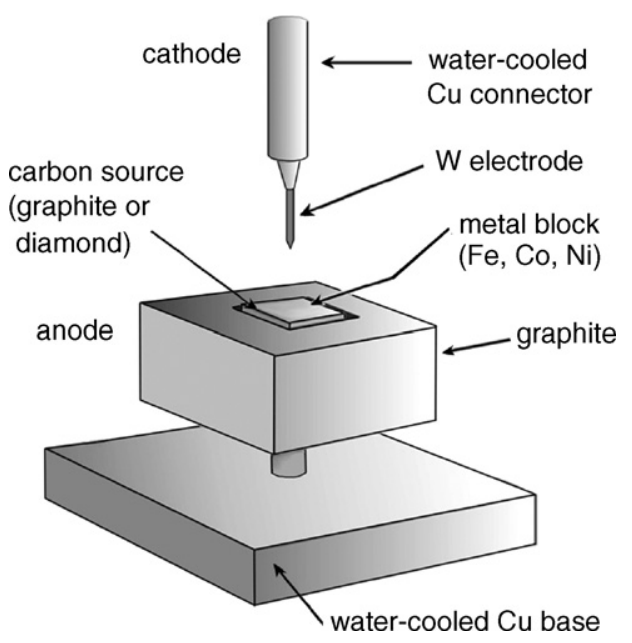

Fig. 1. The arc-discharge setup (schematic).

produce GEM nanoparticles [8]. Although the setup was relatively small, it routinely produced as-made GEM powder at a rate of $3 \mathrm{~g} / \mathrm{h}$.

\subsection{Arc-discharge setup}

As shown in Fig. 1, the carbon source (graphite or diamond powder) and metal blocks $(\mathrm{Fe}, \mathrm{Co}$, or $\mathrm{Ni}$ ) were placed in the graphite crucible as the anode, and a tungsten rod was used as the cathode. When the metal melted at the high temperature in the arc, some graphite (or synthetic diamond powder) dissolved into the metal liquid pool and evaporated simultaneously with the metal. The vapor cooled down once it left the arc, and condensed into the desired nanoparticles. The dc arc current was 100-200 A, the voltage was 20-30 V, and the helium atmosphere was 100-300 Torr.

\subsection{Acid-wash}

To collect well-encapsulated GEM and remove defects, a destructive acidwash method was used. All of the as-made powder was put into a strong acid solution and subjected to ultrasound for $20 \mathrm{~min}$, making sure that all of the defective (not well protected) metal particles dissolved. The weight of the residual powder was then measured to derive the yield percentage of GEM. This process will be the basic method to determine the yield of GEM in this study.

\subsection{Formation mechanism}

To date, only the two-step encapsulation model [4,5] can be used to explain most experimental results. The first step of "phase separation" explains why the turbostratic graphitic shells enclosed a spherical metal nanoparticle. The second step involves the catalytic behavior of several elements, such as iron, nickel, and cobalt. The model predicts that these metals will help amorphous carbon crystallizing into graphite when it is at a high enough temperature. For more detail, please refer to Host et al. [4] and Elliott et al. [5].

\section{Results and discussion}

In earlier work, the production efficiency was greatly improved by using a number of newly developed processes, including: (1) $10 \% \mathrm{CH}_{4} / \mathrm{He}$ mixed gas instead of pure helium, (2) vacuum annealing the as-made GEM powder, and (3) modifying the arc-discharge setup. In this work, by replacing graphite with synthetic diamond powder ( $\sim 150 \mu \mathrm{m}$ in diameter), both the production rate and the recovery ratio were increased significantly.

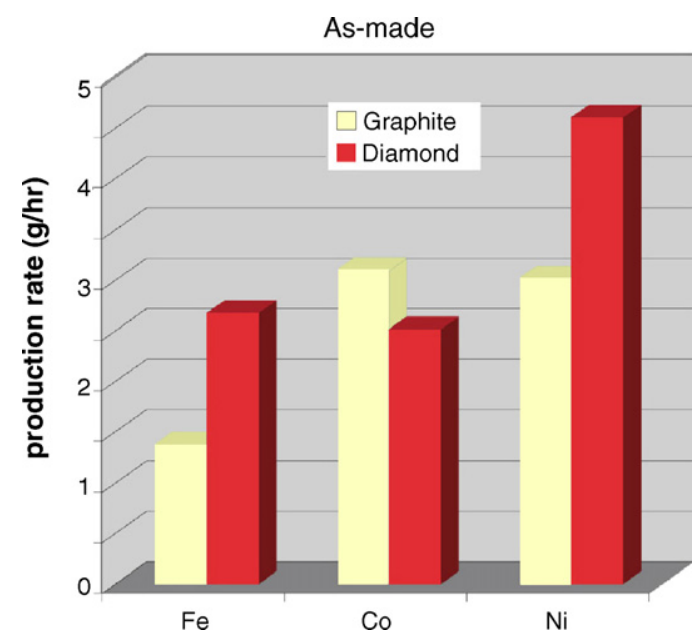

Fig. 2. Average production rates of as-made nanocrystalline powders of $\mathrm{Fe}, \mathrm{Co}$, and $\mathrm{Ni}$ when using graphite and diamond sources.

Fig. 2 shows that the average production rate of the as-made powder increased from $1-3 \mathrm{~g} / \mathrm{h}$ to $2.5-4.5 \mathrm{~g} / \mathrm{h}$, with the exception of cobalt, which showed no increase (probably due to the higher dc current, which was used in our early Co experiments.) One possible explanation is that because the graphitic sheets usual float and cover the surface of the metal liquid pool during arcing, the evaporation area is reduced significantly. Compared to the diamond source metal liquid pool, the graphite source pool has more graphite sheets floating to the surface, thus contributing to a relatively poor production rate.

Fig. 3 shows a significant increase in the recovery ratio (after acid-treatment) from the original average of $7-10 \%$ to $23-33 \%$, thus indicating that diamond is indeed a good carbon source in the production of GEM nanoparticles. However, the graphitic shells of the GEM became thinner (Fig. 4b) and not as well crystallized as when graphite is used as a carbon source (Fig. 4a). In fact, as the graphitic layers cannot be clearly identified, it would be prudent to call the GEM "carbon-encapsulated", instead of "graphite-encapsulated".

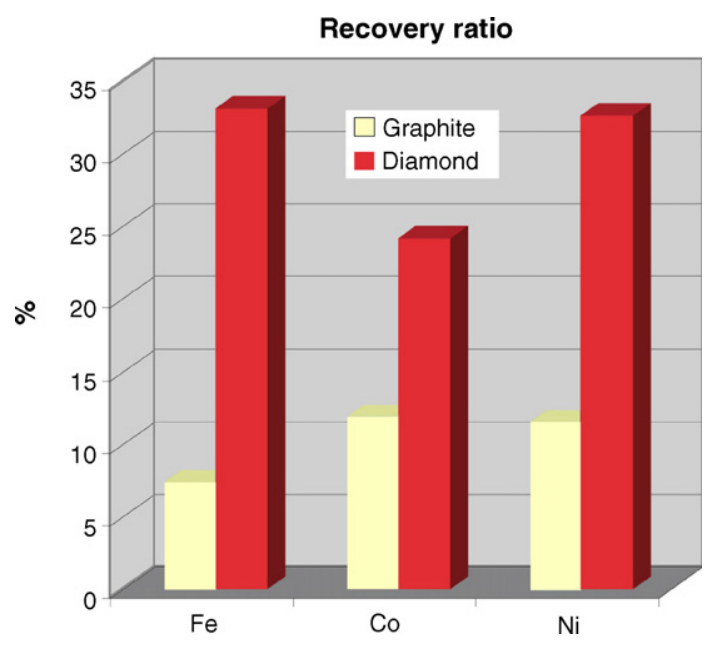

Fig. 3. Average recovery percentages of GEM for $\mathrm{Fe}, \mathrm{Co}$, and $\mathrm{Ni}$ when using graphite and diamond sources. The percentages were derived from the weights of as-made nanocrystalline powders before and after acid-bath treatment. 

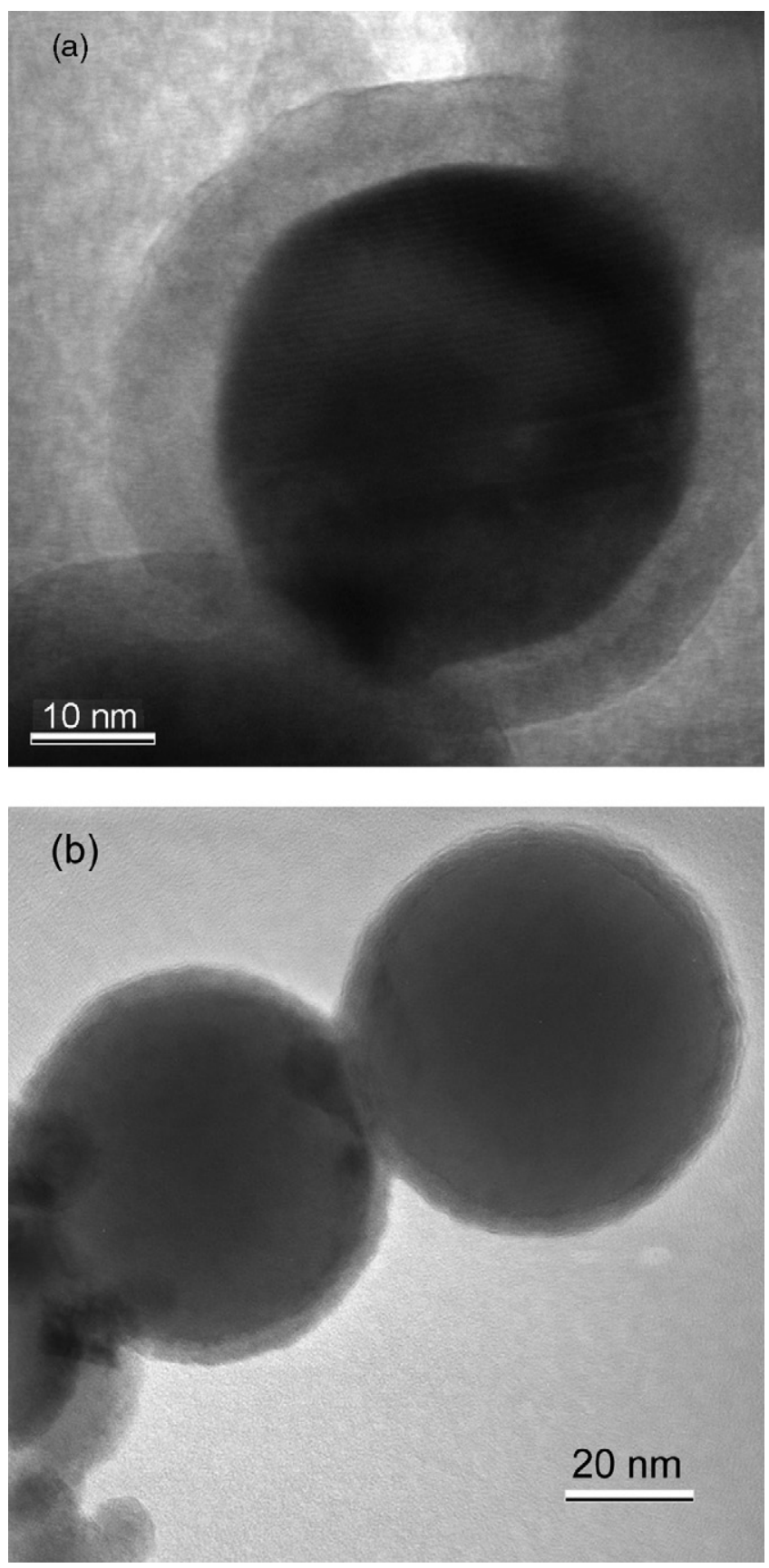

Fig. 4. TEM micrographs (bright field) of Fe-GEM nanoparticles: (a) graphite source and (b) diamond source.

SEM photographs of the cross-sections of the solidified metal blocks after the arcing experiments show that those with a diamond source are full of small graphite flakes (Fig. 5b) and those with a graphite source are mixed with small graphite grains (Fig. 5a) and flakes. The particle size distributions of the graphite flakes found in both types of samples are all log-normal (Fig. 6), which indicates a typical recrystallization and growth process. No diamond was left in the diamond source samples, showing that either the diamond powder had all been dissolved, or had transformed and decomposed into small pieces of graphite. In either case, the process helped to distribute carbon in the liquid pool more homogeneously than in the graphite source samples,
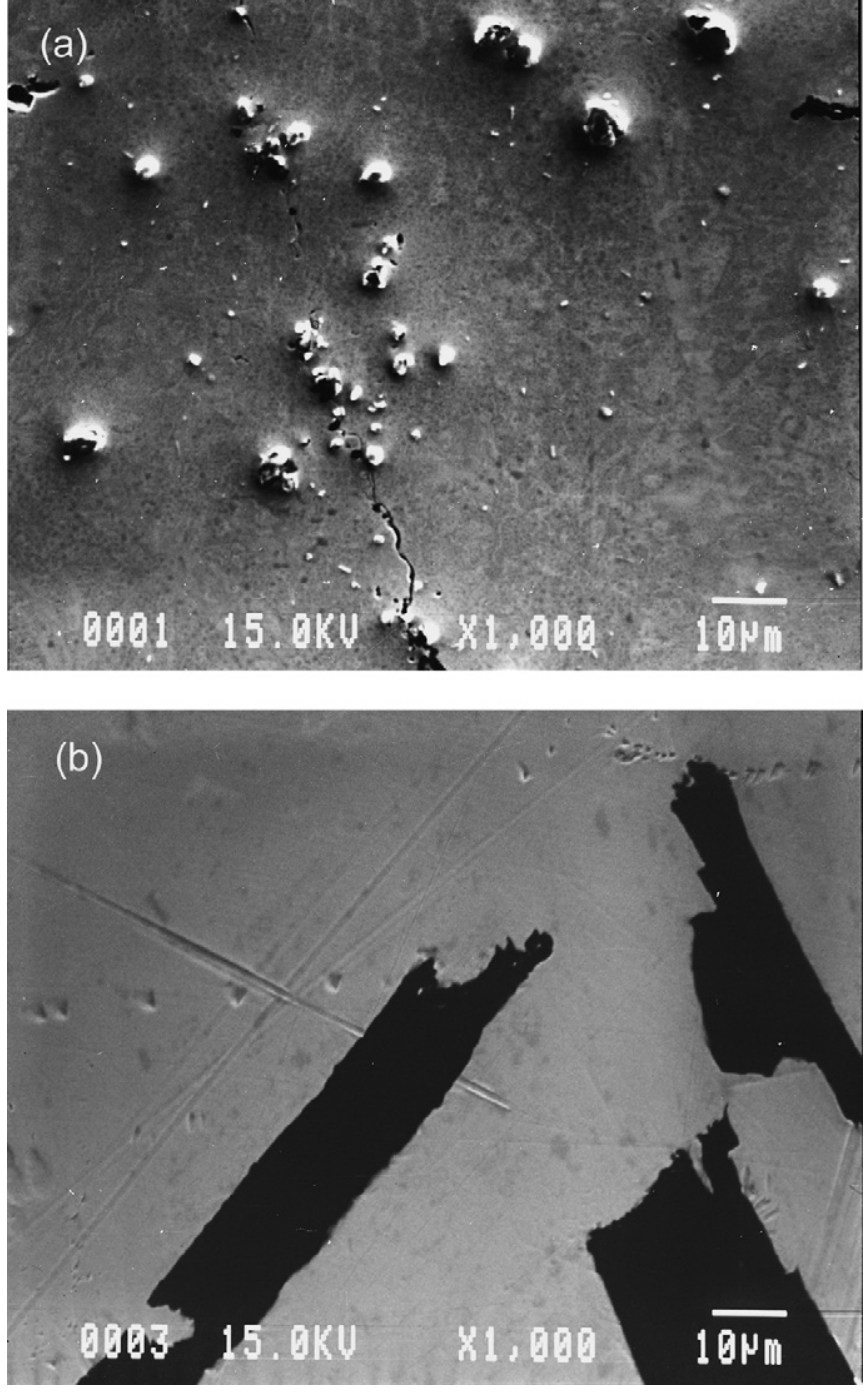

Fig. 5. SEM photographs of the cross-sections of the solidified Ni metal blocks after arcing experiments: (a) diamond source and (b) graphite source.

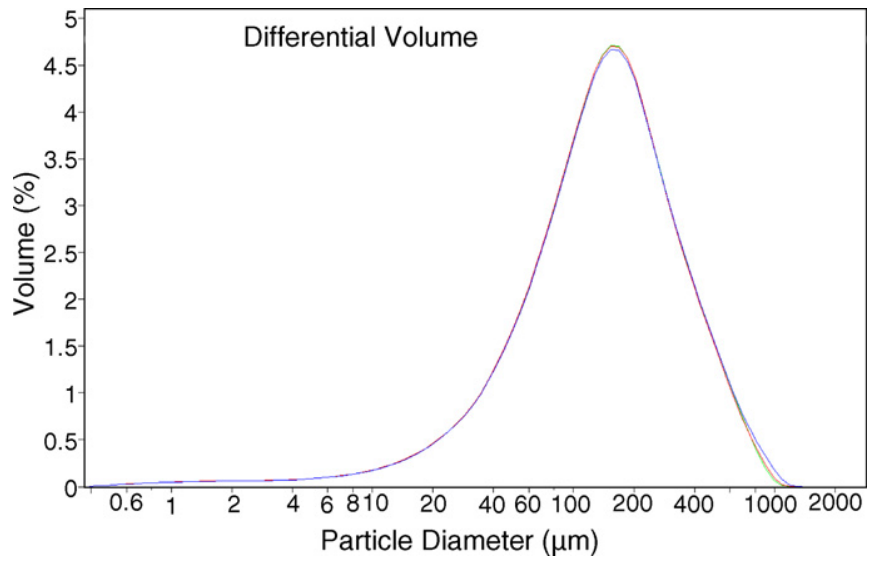

Fig. 6. Typical size distribution curves of the residual graphite flakes deriving from the fully acid-dissolved metal blocks (left in the graphite crucible after the arcing experiments) solution. The size distribution curves of the residual graphite flakes found in both types of samples are all log-normal. 
in which some graphite powder could still be found, probably because graphite is thermodynamically stable.

To determine whether diamond provided more carbon vapor during the synthesis of GEM, TGA analyses of the GEM samples were conducted. For both the graphite and diamond sources, the carbon contents were about the same at $\sim 8 \%$, which indicates that the diamond did not amplify the evaporation of carbon. In addition, analyses of the amount of residual carbon in metal blocks showed that the carbon to metal ratios were about the same at 1:6.5. Therefore, the higher GEM recovery from an acid-bath treatment of a diamond source was obviously not due to a much higher carbon vapor concentration.

What factor improves the encapsulation efficiency of the diamond source samples? If the carbon and metal atoms were equally distributed in the vapor, then the average of $8 \%$ carbon would be more than enough to encapsulate all of the metal nanoparticles. The two-step encapsulation model describes how the small graphitic flakes condense first inside a liquid metal droplet and "float" to the outer surface of the spherical metal; after the collision and coalescence processes every nanoparticles should be well encapsulated. The carbon concentration then probably fluctuates inside the vapor, ensuring that most particles do not have enough carbon to perform "phase-separation" and the encapsulation process. However, most recovered GEM particles from the graphite source (note that there were only $7-10 \%)$ were very well encapsulated, which indicates that they were probably condensed from a vapor area with a high carbon concentration.

As mentioned earlier, the GEM with a diamond source (Fig. 4b) usually had a thinner and not well-crystallized graphitic shell, which could indicate that it was condensed from a less fluctuating carbon concentration vapor so that more nanoparticles could reach the "encapsulation threshold" and form GEM particles.

\section{Conclusions}

Diamond is a very effective carbon source and helps to increase the yields of GEM in all three ferromagnetic metals $\mathrm{Fe}$, $\mathrm{Co}$, and $\mathrm{Ni}$. Although the exact mechanism of this phenomenon is still not clear, experimental evidence shows that diamond, as a metastable phase of carbon, dissolves much more easily in liquid metal pools during arcing than does stable graphite. In comparison to graphite source samples it may help to produce a better-mixed carbon and metal vapor, which will condense into more well encapsulated but thinner-shelled GEM nanoparticles. This work also provides valuable information on the phase transformation of diamond into graphite.

\section{Acknowledgement}

This work was supported in part by National Science Council, Taiwan, Grant NSC93-2116-M-002-037.

\section{References}

[1] V.P. Dravid, J.J. Host, M.H. Teng, B.R. Elliott, J.-H. Hwang, D.L. Johnson, T.O. Mason, J.R. Weertman, Nature 374 (1995) 602.

[2] M.H. Teng, J.J. Host, J.-H. Hwang, B.R. Elliott, J.R. Weertman, T.O. Mason, V.P. Dravid, D.L. Johnson, J. Mater. Res. 10 (1995) 233-236.

[3] J.-H. Hwang, V.P. Dravid, M.H. Teng, J.J. Host, B.R. Elliott, D.L. Johnson, T.O. Mason, J. Mater. Res. 12 (1997) 1076-1082.

[4] J.J. Host, M.H. Teng, B.R. Elliott, J.-H. Hwang, T.O. Mason, J.R. Weertman, D.L. Johnson, V.P. Dravid, J. Mater. Res. 12 (1997) 1268-1273.

[5] B.R. Elliott, J.J. Host, V.P. Dravid, M.H. Teng, J.-H. Hwang, J. Mater. Res. 12 (1997) 3328-3344.

[6] J.J. Host, V.P. Dravid, M.H. Teng, J. Mater. Res. 13 (1998) $2547-$ 2555.

[7] J. Jiao, S. Seraphin, J. Appl. Phys. 83 (1998) 2442-2448.

[8] L.C. Chang, P.Y. Li, J.J. Host, M.H. Teng, Eng. Chem. Metall. (China) 20 (1999) 405-410 (in Chinese). 